\title{
Assessment of health-related quality of life of patients with esophageal squamous cell carcinoma following esophagectomy using EORTC quality of life questionnaires
}

\author{
HONGCHANG SHEN ${ }^{1 *}$, JUE WANG $^{2 *}$, WENHUAN LI ${ }^{1}$, WEIWEI YI $^{1}$ and WEIBO WANG \\ Departments of ${ }^{1}$ Chemotherapy and ${ }^{2}$ Breast and Thyroid Surgery, Shandong Provincial Hospital \\ Affiliated to Shandong University, Jinan, Shandong 250021, P.R. China
}

Received July 16, 2014; Accepted September 3, 2014

DOI: $10.3892 / \operatorname{mco} .2014 .434$

\begin{abstract}
Esophageal cancer is one of the leading causes of cancer-related mortality and surgery is currently the main treatment modality for resectable esophageal cancer. To assess health-related quality of life (HRQL) of patients with esophageal squamous cell carcinoma (ESCC) following esophagectomy, 62 consecutive patients with middle ESCC were randomly assigned into hand video-assisted thoracoscopic surgery (HVATS) $(n=33)$ and Ivor-Lewis surgery (ILS) $(n=29)$ groups. Quality of life questionnaires (QLQ)-C30 and QLQ-OES18, published by the European Organization for Research and Treatment of Cancer, were used prior to treatment and at regular intervals until 6 months following surgery. The results of QLQ-C30 and QLQ-OES18 demonstrated that i) patients with comorbidities and advanced tumor stage (III-IV) exhibited increased risk of poor HRQL, while their gender, age, body mass index and anastomosis location were not associated with HRQL at 6 months after surgery; ii) all the patients had worse functional, symptom and global scores within 6 months after surgery; iii) patients in the HVATS group had similar baseline functional and symptom scores to those of patients in the ILS group; however, their functional and global scores were higher and their symptom scores were lower compared to those of patients in the ILS group; iv) the HRQL of patients in the HVATS group returned to preoperative levels within a shorter time period compared to patients in the ILS group. There were significant differences in global
\end{abstract}

Correspondence to: Professor Weibo Wang, Department of Chemotherapy, Shandong Provincial Hospital Affiliated to Shandong University, 324 Jingwu Road, Jinan, Shandong 250021, P.R. China

E-mail: wbwb1620@163.com

${ }^{*}$ Contributed equally

Key words: esophageal neoplasms, quality of life, hand video-assisted thoracoscopic surgery, esophagectomy health, physical functioning, fatigue and pain scales between the two groups. In QLQ-OES18, the dysphagia and gastroesophageal reflux scales were improved in both the HVATS and ILS groups, but no significant differences were observed between the two groups. In addition, the overall survival rate was similar in the two groups. Taken together, our findings indicated that HVATS is a safe procedure, associated with less disturbance to short-term HRQL compared to ILS. Therefore, it appears reasonable to select HVATS for patients with early-stage middle esophageal cancer.

\section{Introduction}

Esophageal cancer is one of the leading causes of cancer-related mortality in several countries. It is estimated that 200,000 individuals succumb to esophageal cancer annually $(1,2)$. Surgery is the main treatment modality for resectable esophageal cancer (3). However, conventional surgery is associated with higher mortality and postoperative complication rates (4). In the past, patients reported more concerns regarding the severe postoperative complications and survival time, but appeared to be less concerned with the overall quality of life.

An increasing number of minimally invasive surgical techniques are currently used to reduce the incidence of postoperative complications and the mortality rate of patients with esophageal cancer $(5,6)$. However, it is difficult to undertake such surgery without video assistance. Video-assisted thoracic surgery (VATS) has become the mainstay of minimally invasive thoracic surgery, as it is reliable, minimizes trauma and suffering and allows for a shorter recovery time $(6,7)$. The combination of VATS and conventional thoracotomy may be used to complete the removal of lesions at an earlier stage and, thus, has become the standard method for lymph node dissection. In 2002, the Shandong Provincial Hospital Affiliated to Shandong University, developed a novel technique, namely hand video-assisted thoracoscopic surgery (HVATS), involving a surgeon operating by hand through an abdominal incision into the chest with the assistance of thoracoscopy. This method successfully combined minimally invasive and conventional surgery $(8,9)$.

The main objective of this study was to assess the effect of HVATS and Ivor-Lewis surgery (ILS) on short-term 
Table I. Clinical characteristics of patients with esophageal cancer.

\begin{tabular}{|c|c|c|c|c|}
\hline \multirow[b]{2}{*}{ Characteristics } & \multicolumn{2}{|c|}{ Total no. of patients $(\mathrm{n}=62)$} & \multirow[b]{2}{*}{ Chi-square } & \multirow[b]{2}{*}{ P-value } \\
\hline & $\begin{array}{c}\text { HVATS } \\
(\mathrm{n}=33)\end{array}$ & $\begin{array}{l}\text { Ivor-Lewis } \\
\quad(\mathrm{n}=29)\end{array}$ & & \\
\hline Gender & & & 0.111 & 0.739 \\
\hline Male & 25 & 23 & & \\
\hline Female & 8 & 6 & & \\
\hline Age, years & & & 0.295 & 0.587 \\
\hline$<60$ & 10 & 7 & & \\
\hline$\geq 60$ & 23 & 22 & & \\
\hline Body mass index at surgery & & & 0.083 & 0.773 \\
\hline$<24.9$ & 17 & 16 & & \\
\hline$\geq 25$ & 16 & 13 & & \\
\hline Comorbidities & & & 0.009 & 0.924 \\
\hline No & 11 & 10 & & \\
\hline Yes & 22 & 19 & & \\
\hline \multicolumn{5}{|l|}{ Tumor location } \\
\hline Upper and middle & 33 & 29 & & \\
\hline Lower & 0 & 0 & & \\
\hline \multicolumn{5}{|l|}{ Histology } \\
\hline Adenocarcinoma & 0 & 0 & & \\
\hline Squamous cell carcinoma & 33 & 29 & & \\
\hline Anastomosis location & & & 10.452 & 0.001 \\
\hline Neck & 33 & 21 & & \\
\hline Upper right chest & 0 & 8 & & \\
\hline \multicolumn{5}{|l|}{ Substitute } \\
\hline Stomach & 33 & 29 & & \\
\hline Jejunum & 0 & 0 & & \\
\hline TNM stage & & & 0.779 & 0.854 \\
\hline 0 & 4 & 3 & & \\
\hline I & 18 & 17 & & \\
\hline II & 10 & 7 & & \\
\hline III & 1 & 2 & & \\
\hline \multicolumn{5}{|l|}{ IV } \\
\hline Complications & & & 0.827 & 0.363 \\
\hline No & 23 & 17 & & \\
\hline Yes & 10 & 12 & & \\
\hline
\end{tabular}

HVATS, hand video-assisted thoracoscopic surgery.

health-related quality of life (HRQL) of patients with esophageal cancer using the quality of life questionnaires (QLQ)-C30 and QLQ-OES18, published by the European Organization for Research and Treatment of Cancer (EORTC), in order to determine the application perspective of HVATS in the treatment of esophageal cancer.

\section{Materials and methods}

Patients. A total of 62 patients with pathologically confirmed, newly diagnosed middle esophageal cancer, who underwent surgery at the Shandong Provincial Hospital Affiliated to Shandong University between January, 2005 and June, 2007, were enrolled in this study. The eligibility criteria included Karnofsky performance score $\geq 70$, age $18-75$ years, no adjuvant therapy, no distant metastases, survival for $>3$ months after surgery, follow-up of $\geq 5$ years after surgery and no causes of death other than esophageal cancer.

This study was approved by the Ethics Committee of the Shandong Provincial Hospital Affiliated to Shandong University and written informed consent was obtained from all the participating subjects. 
Table II. Associations between clinical characteristics and quality of life questionnaire-C30 aspects of health-related quality of life.

\begin{tabular}{|c|c|c|c|c|c|c|c|c|c|c|}
\hline \multirow[b]{2}{*}{ Characteristics } & \multicolumn{2}{|c|}{ GQL } & \multicolumn{2}{|c|}{ Physical function } & \multicolumn{2}{|c|}{ Role function } & \multicolumn{2}{|c|}{ Emotional function } & \multicolumn{2}{|c|}{ Social function } \\
\hline & OR & $95 \% \mathrm{CI}$ & OR & $95 \% \mathrm{CI}$ & OR & $95 \%$ CI & OR & $95 \%$ CI & OR & $95 \% \mathrm{CI}$ \\
\hline \multicolumn{11}{|l|}{ Gender } \\
\hline Male ${ }^{\mathrm{a}}$ & 1 & & 1 & & 1 & & 1 & & 1 & \\
\hline Female & 1.24 & $0.54-1.97$ & 1.12 & $0.53-1.74$ & 0.68 & $0.41-1.29$ & 0.99 & $0.57-1.79$ & 0.69 & $0.36-1.68$ \\
\hline \multicolumn{11}{|l|}{ Age, years } \\
\hline$<60^{\mathrm{a}}$ & 1 & & 1 & & 1 & & 1 & & 1 & \\
\hline$\geq 60$ & 0.61 & $0.38-1.23$ & 1.03 & $0.57-1.88$ & 0.59 & $0.31-1.42$ & 0.61 & $0.33-0.97$ & 0.54 & $0.41-1.01$ \\
\hline \multicolumn{11}{|l|}{ BMI } \\
\hline$<24.9^{\mathrm{a}}$ & 1 & & 1 & & 1 & & 1 & & 1 & \\
\hline$\geq 25$ & 0.84 & $0.71-1.03$ & 0.99 & $0.86-1.32$ & 0.88 & $0.73-1.25$ & 0.9 & $0.66-1.13$ & 0.73 & $0.59-0.97$ \\
\hline \multicolumn{11}{|l|}{ Comorbidities } \\
\hline $\mathrm{No}^{\mathrm{a}}$ & 1 & & 1 & & 1 & & 1 & & 1 & \\
\hline Yes & 1.49 & $0.97-2.52$ & 1.79 & $1.21-2.83$ & 1.86 & $1.07-2.89$ & 1.61 & $0.87-3.01$ & 1.55 & $0.92-2.34$ \\
\hline \multicolumn{11}{|l|}{ TNM stage } \\
\hline $0-\mathrm{I}^{\mathrm{a}}$ & 1 & & 1 & & 1 & & 1 & & 1 & \\
\hline II & 1.45 & $0.88-2.71$ & 1.32 & $0.68-2.70$ & 1.88 & $0.96-3.73$ & 1.27 & $0.61-2.08$ & 1.53 & $0.69-2.66$ \\
\hline III-IV & 1.71 & $0.73-2.91$ & 1.58 & $0.81-3.01$ & 1.98 & $1.17-3.92$ & 1.54 & $0.69-2.68$ & 1.34 & $0.58-2.36$ \\
\hline
\end{tabular}

${ }^{a}$ These variables were seen as references. GQL, global quality of life; OR, odds ratio; CI, confidence interval; BMI, body mass index.

Surgical techniques

HVATS. A total of 33 patients underwent HVATS under general anesthesia, positioned on the right side of the body and rotated $45^{\circ}$. The key steps of HVATS included i) exploration and mobilization of the stomach through an upper median abdominal incision; ii) mobilizing the esophageal tumor extraperitoneally using the left hand of the operator from the right side of the abdominal incision under the costal arch; iii) mobilizing the esophagus from the top of chest in the rear to the arch of the azygos vein and drawing it from the left neck incision; iv) performing lymph node dissection; and v) pulling the stomach into the chest through the diaphragmatic hiatus and anastomosing with the esophagus end-to-side under surveillance through the neck incision.

ILS. A total of 29 patients underwent ILS under general anesthesia, positioned on the right side of the body and rotated $45^{\circ}$. The key steps of ILS included i) exploring and mobilizing the esophagus through a chest incision at the right 4th intercostal space; ii) mobilizing the stomach through an upper median abdominal incision; iii) performing lymph node dissection; and iv) anastomosing with the esophagus end-to-side at the neck or right thoracic cupola.

Quality of life evaluation. All the patients completed the QLQ-C30 and QLQ-OES18 published by EORTC. EORTC QLQ-C30 (version 3.0) is a self-assessment questionnaire describing 5 functional indices, including physical, role, cognitive, emotional and social functions, 3 symptom indices, including fatigue, pain and nausea or vomiting and 1 general score. EORTC QLQ-OES18 includes 18 indices, namely specific symptoms, such as dysphagia, esophageal reflux and bucking. EORTC QLQ-C30 was believed to be of superior validity and reliability, as assessed by Hjermstad (10) in 190 untreated patients. The combination of QLQ-C30 and QLQ-OES18 is considered to precisely reflect the changes in the quality of life of esophageal cancer patients and is recommended by EORTC to evaluate the quality of life of such patients.

Survey methods. An examination was conducted 1 week prior to surgery and at 2, 4, 12 and 24 weeks after surgery. All the questionnaires were completed by the patients themselves. Higher functional and general scores of EORTC QLQ-C30 indicate a better quality of life, whereas higher symptom scores of EORTC QLQ-C30 and QLQ-OES18 reflect a worse quality of life.

Statistical analysis. Data are expressed as means \pm standard deviation and were compared by the Chi-square test. The statistical significance of the differences in the questionnaire scores between the two groups was determined by the Student's t-test. The logistic regression model was used to assess the associations between preoperative clinical characteristics and HRQL scores at 6 months after surgery. The Chi-square test was used to evaluate the correlation between the selection of surgical technique and clinicopathological characteristics. The Kaplan-Meier survival curve was applied to assess the 5-year survival rate and the log-rank test was used to evaluate the differences in survival distributions. $\mathrm{P}<0.05$ was considered to indicate a statistically significant difference. 
A

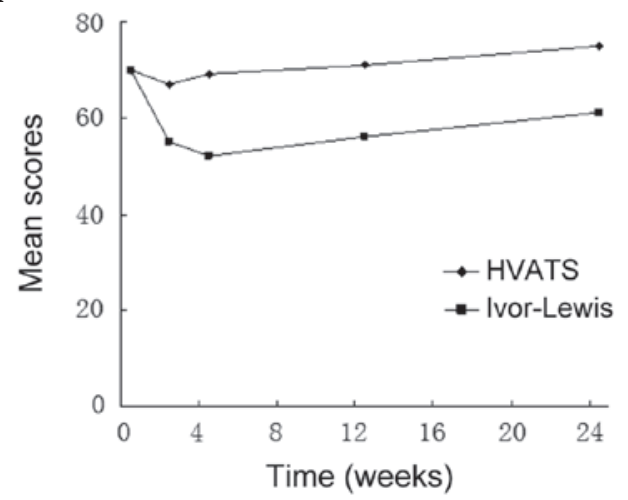

B

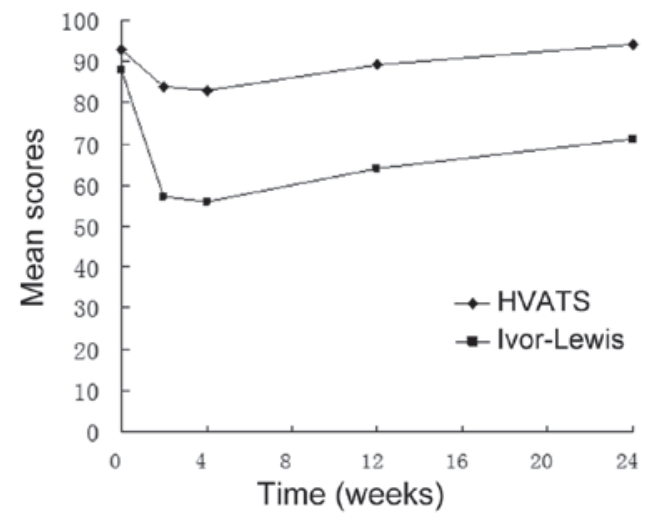

C

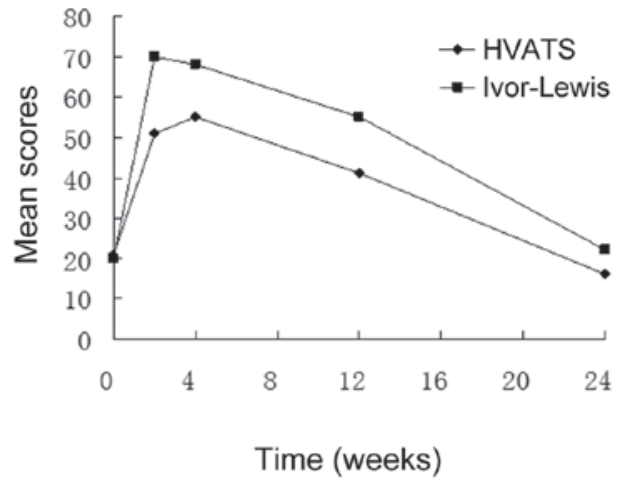

D

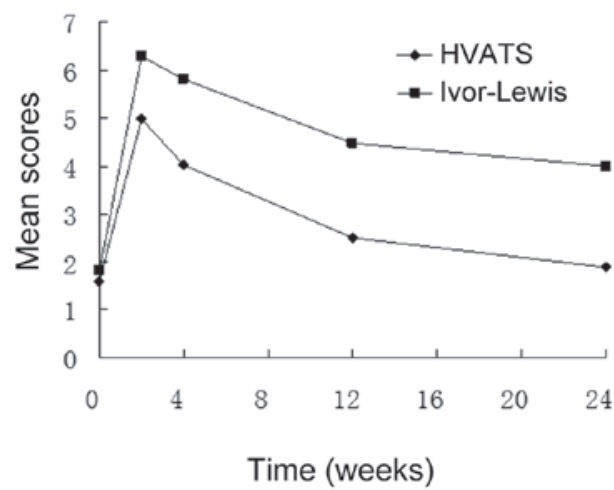

Figure 1. Comparison of hand video-assisted thoracoscopic surgery (HVATS) with Ivor-Lewis surgery according to the European Organization for Research and Treatment of Cancer (EORTC) quality of life questionnaire (QLQ)-C30. (A) General scores. (B) Physical scores. (C) Fatigue scores. (D) Pain scores.

A

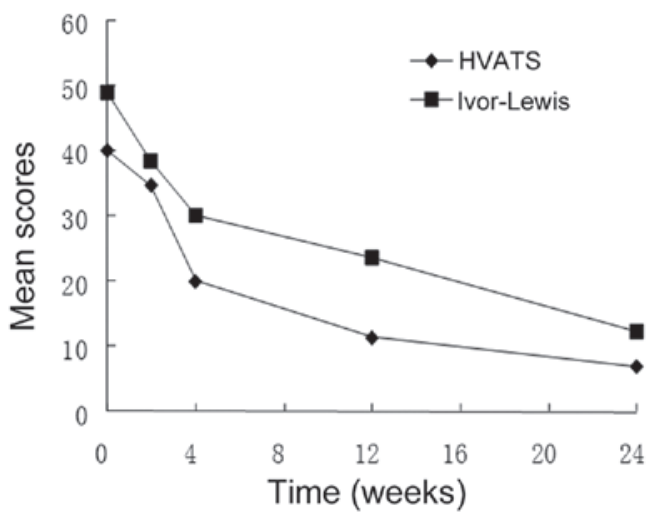

B

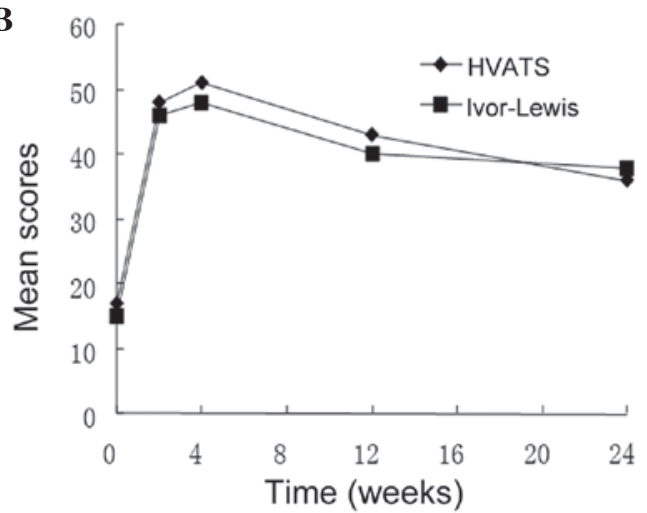

Figure 2. Comparison of hand video-assisted thoracoscopic surgery (HVATS) with Ivor-Lewis surgery according to the European Organization for Research and Treatment of Cancer (EORTC) quality of life questionnaire (QLQ)-OES18. (A) Dysphagia scores. (B) Esophageal reflux scores.

\section{Results}

Clinical characteristics. The patient characteristics, grouped by surgical procedures, are shown in Table I. The Chi-square test revealed no significant differences in gender, age, body mass index (BMI), substitute, TNM stage and complications among the 62 patients. However, anastomosis location was significantly different between patients in the HVATS and ILS groups.

Associations between clinical characteristics and HRQL. The 62 patients were analyzed for the associations between clinical characteristics and QLQ-C30 functional aspects of HRQL
6 months following esophagectomy. The results revealed that gender and BMI were not significantly associated with the functional aspects of HRQL at 6 months after surgery, whereas the presence of comorbidities was significantly associated with poor physical and role function scores (Table II). In addition, patients aged $<60$ years exhibited poorer emotional function compared to patients aged $>60$ years and patients with advanced tumor stage (III-IV) exhibited an increased risk of poor role function (Table II).

Quality of life. A total of 282 questionnaires were qualified (282/296). According to the EORTC QLQ-C30, the general, 
physical, fatigue and pain scores were significantly different between the HVATS and ILS groups. However, the remaining scores of EORTC QLQ-C30 and all the scores of EORTC QLQ-OES18 were not significantly different between the two groups. The changes in all the mean scores are presented in Figs. 1 and 2.

The portrait of the changes of EORTC QLQ-C30 revealed that the preoperative symptom and functional scores were similar between the two groups. However, compared to the preoperative scores, the functional scores at 2 weeks postoperatively, such as general and physical scores, were decreased, while the symptom scores, such as pain and fatigue scores, were significantly increased, particularly in the ILS group (Fig. 1). In addition, the functional scores of the patients in the HVATS group had partially recovered at 3 months and had reached preoperative level at 6 months. Although the functional scores of patients in the ILS group gradually increased during the first 3 months postoperatively, they remained significantly lower compared to the preoperative levels at 6 months (Fig. 1). The changes in the EORTC QLQ-OES18 scores demonstrated that the postoperative dysphagia scores of the patients in both groups were significantly lower compared to their preoperative scores, reflecting the success of the surgical intervention. However, the scores of dysphagia, esophageal reflux and other specific symptoms of the two groups were not significantly different prior to and after surgery ( $\mathrm{P}>0.05)$ (Fig. 2).

Furthermore, the general quality of life scores of the patients in the HVATS group were significantly higher compared to those of the patients in the ILS group at all the monitoring points $(\mathrm{P}<0.05)$.

\section{Discussion}

There are several surgical techniques applied to the treatment of esophageal cancer, although their survival rates are not significantly different. The quality of life as 1 of the 3 endpoints for cancer therapy evaluation has attracted extensive attention over the last few years. Blazeby et al (11) evaluated the quality of life of esophageal cancer patients who received surgery or palliative treatment using EORTC QLQ-C30 and observed that the quality of life of the former was significantly higher compared to that of the latter, suggesting that surgical treatment may be a preferred choice for patients with esophageal cancer. Studies on the effect of VATS on the quality of life are currently more focused on patients with lung cancer $(12,13)$. To the best of our knowledge, this is the first study on the effect of VATS on the quality of life of patients with esophageal cancer.

HVATS improved the general short-term quality of life. This study demonstrated that the general quality of life scores of patients in the two groups were significantly higher in the HVATS group compared to those in the ILS group at all the monitoring points. This may be mainly attributed to the fact that HVATS minimizes the injury to the chest wall, preserves lung function and relieves postoperative pain; therefore, patients may find it easier to exercise and cough following surgery compared to ILS. Thus, HVATS may be conducive to recovery and improved overall quality of life.

HVATS may reduce postoperative fatigue and restrictions to physical functionality. A high proportion of patients exhibited fatigue and decreased locomotor activity in the two groups at all the monitoring time points. During the first 3 months, the patients in the HVATS group had higher physical and lower fatigue scores compared to those of patients in the ILS $(\mathrm{P}<0.05)$, possibly due to the fact that HVATS was associated with less extensive injury to the chest wall and was conducive to the recovery of physical functions.

Pain is one of the major causes of discomfort, which may compromise rest, breathing and coughing and may also cause pneumonia, atelectasis and other complications. This study demonstrated that, at all the monitoring points, the patients in the HVATS group had lower pain scores compared to patients in the ILS group, indicating that HVATS was associated with significantly less pain $(\mathrm{P}<0.05)$. HVATS, which only requires a small incision and does not require cutting through the latissimus dorsi and anterior serratus muscles or a rib retractor, may help avoid injuries to the ribs, intercostal nerves and vertebral joints, thereby minimizing postoperative pain. It was previously demonstrated that thoracoscopic surgery reduces postoperative pain and the amount of postoperative analgesics administered (14).

Although HVATS is associated with certain advantages regarding the functional scores mentioned above, the EORTC QLQ-OES18 symptomatic scores, such as dysphagia, gastroesophageal reflux and diarrhea, were not significantly different between patients in the HVATS and ILS groups $(\mathrm{P}>0.05)$.

In conclusion, HVATS for esophageal cancer patients significantly improved the short-term quality of life compared to ILS. However, due to the limited case number, the data may be flawed and the conclusions may come with certain limitations. In addition, the effect of HVATS on long-term quality of life has not been clearly determined. Thus, further prospective studies, including larger patient samples, are required to verify our findings.

\section{Acknowledgements}

All the authors listed have approved the manuscript. All the procedures were conducted in accordance with the ethical standards of the Committee on Human Experimentation of Shandong University. This study was supported by the Science and Technology Foundation of Shandong Province (grant no. 2013GSF11845) and the Science and Technology Project of Jinan (grant no. 201202023).

\section{References}

1. Jemal A, Siegel R, Ward E, Hao Y, Xu J, Murray T and Thun MJ: Cancer statistics, 2008. CA Cancer J Clin 58: 71-96, 2008.

2. Gallo A and Cha C: Updates on esophageal and gastric cancers. World J Gastroenterol 12: 3237-3242, 2006.

3. Enzinger PC and Mayer RJ: Esophageal cancer. N Engl J Med 349: 2241-2252, 2003.

4. Fabian T, Martin JT, McKelvey AA and Federico JA: Minimally invasive esophagectomy: a teaching hospital's first year experience. Dis Esophagus 21: 220-225, 2008.

5. Shichinohe T, Hirano S and Kondo S: Video-assisted esophagectomy for esophageal cancer. Surg Today 38: 206-213, 2008.

6. Sunpaweravong S, Chewatanakornkul S and Ruangsin S: Initial experience and result of thoracoscopic and laparoscopic esophagectomy. J Med Assoc Thai 91: 1202-1205, 2008.

7. Toker A, Eroglu O, Ziyade S, Tanju S, Senturk M, Dilege S and Kalayci G: Comparison of early postoperative results of thymectomy: partial sternotomy vs. videothoracoscopy. Thorac Cardiovasc Surg 53: 110-113, 2005. 
8. Du JJ, Meng L, Chen JH, Peng ZM, Wang L, Zhang L and Wang XH: Hand-assisted video-thoracoscopy for resecion of esophageal cancer. Chin J Surg 43: 351-353, 2005 (In Chinese).

9. Du JJ, Meng L, Chen JH, Peng ZM, Wang L, Zhang L and Wang XH: Clinical therapeutic effect of esophageal carcinoma with hand video assisted surgery. Chin J Surg 45: 822-824, 2007 (In Chinese).

10. Hjermstad MJ1, Fossa SD, Bjordal K and Kaasa S: Test/retest study of the European Organization for Research and Treatment of Cancer Core Quality-of-Life Questionnaire. J Clin Oncol 13: 1249-1254, 1995.

11. Blazeby JM, Williams MH, Brookes ST, et al: Quality of life measurement in patients with oesophageal cancer. Gut 37: 505-508, 1995.
12. Nakata M, Saeki H, Yokoyama N, et al: Pulmonary function after lobectomy: video-assisted thoracic surgery versus thoracotomy. Ann Torac Surg 70: 938-941, 2000.

13. Walker WS, Codispoti M, Soon SY, et al: Long-term outcomes following VATS lobectomy for non-small cell bronchogenic carcinoma. Eur J Cardiothorac Surg 23: 397-402, 2003.

14. Paiva JM and Wright GM: Hand-assisted thoracoscopic surgery causes less postoperative pain than limited thoracotomy after cessation of epidural analgesia. Heart Lung Circ 13: 374-378, 2004. 